\section{Schering STAR Program}

$\mathrm{T}$ ing was the first of its kind in South Africa and was a resounding success. STAR is an acronym for "Schering Training and Advances in Radiology". The arrangements are unique in that there is a single sponsor and there are no marketing or technical exhibits. The program was presented over two days in Johannesburg (29-30 August) and Cape Town (2-3 September.) The academic and organizational standards were very high. The meetings were well attended by radiologists, registrars and a smattering of clinicians.

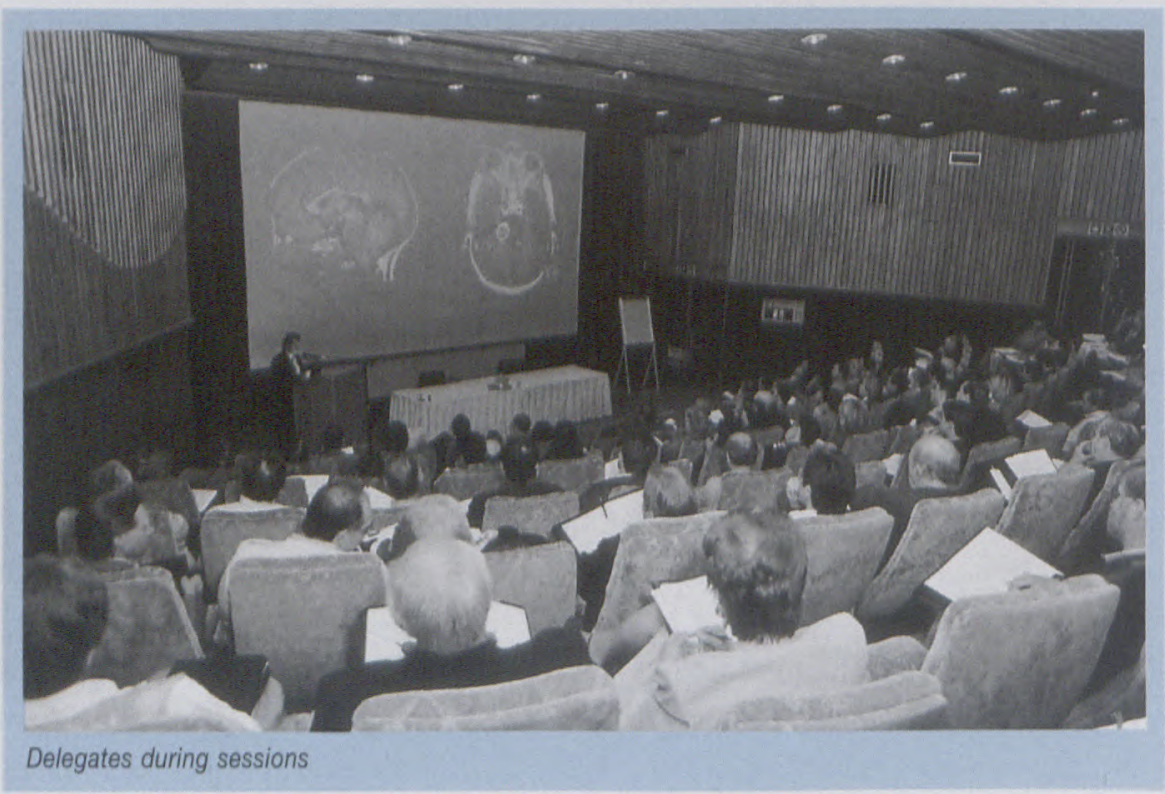

The speakers were superb: eloquent, lucid and a pleasure to listen to. We were privileged to hear Hedvig Hricak, Alexander Margulis and Roy Filly (all UCSF), Elias Zerhouni (Baltimore), Hans Schild (Bonn) and
Francis Brunelle (Paris.) The topics were well selected and included a comprehensive mix of disciplines. The emphasis was on quality and value, and the content included practical refinements to current practice as well as indicators of future trends.

\section{Paediatric neuroradiology}

Francis Brunelle presented an excellent practical overview of the imaging of brain tumours in paediatric patients, providing useful management guidelines. He reminded the audience that these relatively common tumours predominate below the tent in children, typically presenting with raised intracranial pressure. He spent some time on sedation techniques. In one point, he suggested that if a number of children required imaging, one could sedate them simultaneously, and select the most sedated child for each scan. It may be sensible to continue onto surgery if a GA is required for MRI.

Although CT is helpful, MRI is the primary imaging modality in these patients, as well as for stereotaxis and follow-up. Angiography is reserved for preoperative embolisation. He then discussed the posterior fossa and supratentorial tumours sequentially, and mentioned some of the uncommon seizure-related presentations.

He also discussed interventional neuroradiology in children, and addressed imaging equipment needs and recent catheter developments. $\mathrm{He}$ briefly discussed and provided some useful tips in catheter manipulation, such as the use of two $3 \mathrm{~F}$ catheters without larger guiding catheters, and the technique of superselective cannulation with flow-directed 
from page 6

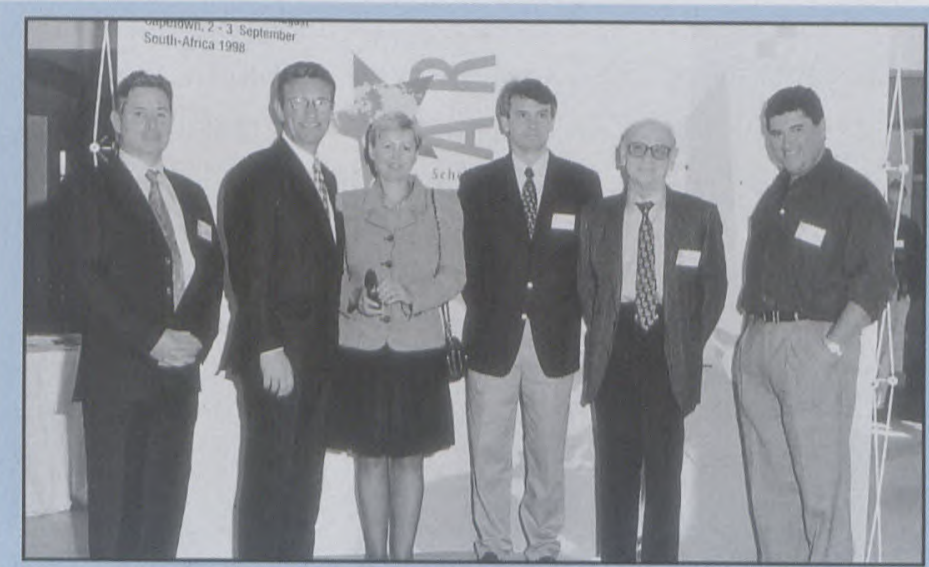

The faculty (left to right): Prof F Brunelle, Prof R Filly, Prof H Hricak, Prof $H$ Schild, Prof A Margulis, Prof E Zerhouni s u g g e s t ed focussing one's attention on masses in the 25-60 $\mathrm{mm}$ range. His first thought is generally - will this mass disappear? Secondly, is it unilocular without mural nodules? Thirdly, is it suspicious microcatheters. He paid individual attention to embolisation of arteriovenous malformations (usually exclusively worked up by MRI), vein of Galen aneurysms, intraventricular meningiomas and the external carotid territory.

He concluded by describing the modern imaging of paediatric hydrocephalus, emphasising the dynamic flow patterns throughout the cisterns of the CNS. The various causes of hydrocephalus were outlined. Accurate measures of ventricular volume are possible. The role of endoscopic surgical ventriculocisternostomy was described, including the imaging evaluation of its patency. Interestingly, so-called external hydrocephalus he felt is in reality due to chronic bilateral subdural effusions, as CT myelography reveals only contrast in the central CSF displaced away from the peripheral chronic subdural collections.

\section{Pelvic ultrasound}

Roy Filly took on ovarian masses in a very practical and entertaining overview during which he emphasised a very directed approach. Considering that any mass over $6 \mathrm{~cm}$ will most likely be excised, while any mass under $25 \mathrm{~mm}$ is most likely normal, he

with any of septations, mural or septal nodules, thick irregular wall or solid lesions? The specific presence of bright echoes and shadowing echodensities should suggest the diagnosis of a teratoma. These bright echoes may represent a fat nodule, a ball of hair, tooth or bone. The e $\mathrm{nd} \mathrm{om} \mathrm{e} \mathrm{-}$ trioma he labelled the great fooler of all gynaecological ultrasound. Their variable appearance and the utility of
MRI in diagnosing these cysts was emphasised. The poor performance of Doppler in predicting the nature of ovarian lesions was also noted, although it is useful in separating clot from tumour.

He then gave a very helpful approach to polyhydramnios. The peak volume of amniotic fluid is at 34 weeks, ranging from 500 to $1500 \mathrm{ml}$. The relative amniotic fluid to fetal

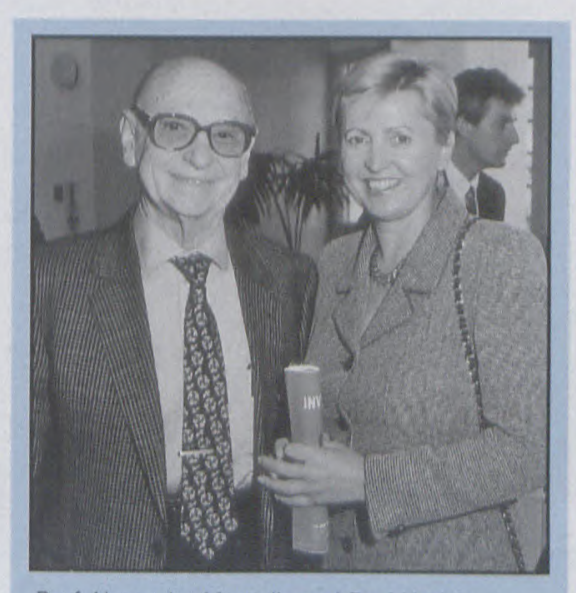

Prof Alexander Margulis and Prof Hedvig Hricak, with Armin Junkuhn from Schering in the background

ratio is maximal at 20-24 weeks. The means of diagnosing polyhydramnios were covered in detail. Fifty percent of anomalous fetuses have increased amniotic fluid volume. The urgency of the diagnosis of oligohydramnios was emphasised.

Thereafter the tricky ultrasonic evaluation of the fetal cerebral ventricle was simplified by focussing on the dimension of the ventricular atrium (defined by the site where the choroid turns), that remains remarkably constant in its luminal diameter throughout and after gestation. The predictive value of atrial diameter was excellent, with an $88 \%$ rate of fetal anomalies when the atrial diameter exceeded $10 \mathrm{~mm}$. The ideal measurement time is $18-20$ weeks.

Twins were addressed, with again a down-toearth approach highlighting the differentiation of mono- and dichorionic twins and the twin transfusion syndrome.

\section{Interventional radiology}

Hans Schild tackled the interventive radiology component of the workshop. He covered the biological effects of metallic

stent implantations, concentrating on the risks of thrombosis (related to damage, material thrombogenicity, flow and shear stresses) and neointimal hyperplasia. Pain therapy by means of regional drug administration, arterial embolisation, ureteric embolisation, tumour drainage and CT-guided coeliac, splanchnic and superior hypogastric nerve blocks were described. CT infiltrations into 


\section{from page 7}

the skeletal system were also covered. Uroradiological interventions dealt with renal artery angioplasty and stenting, spermatic vein occlusion, penile vein occlusion and nephrostomy. Ureteric dilatation and stenting, plus ureteric embolisation and transurethral balloon dilatation of the prostate completed the section.

\section{Chest CT}

Elias Zerhouni conducted a beautifully methodical walk through high resolution $\mathrm{CT}$, explaining all the appearances encountered on the basis of the 300-600 micron pixel resolution of high resolution CT. He covered the reasons behind the appearances of ground glass opacities, interface signs, reticulation, honeycombing, nodules and cystic changes. Specific entities included idiopathic pulmonary fibrosis, asbestosis, silicosis, lymphangitis carcinomatosa, sarcoidosis, histiocytosis $\mathrm{X}$, pulmonary lymph-

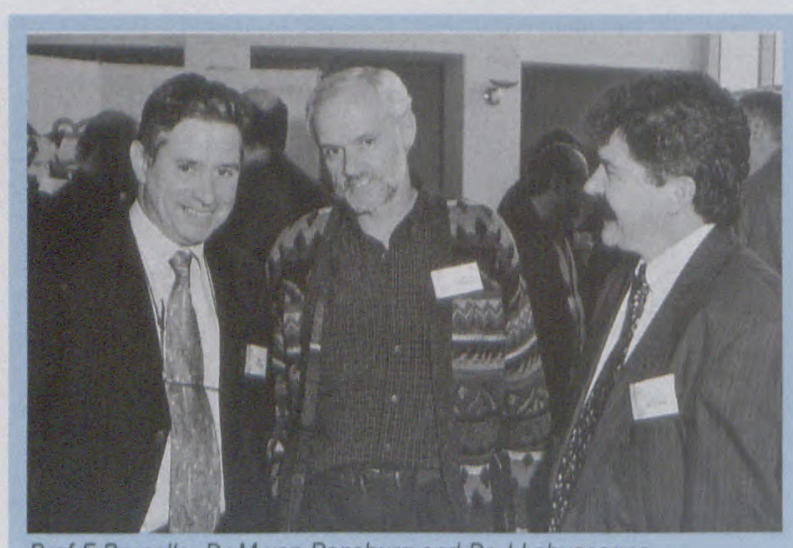

Prof F Brunelle, Dr M van Rensburg and Dr J Labuscagne

angioleiomyomatosis, emphysema, BOOP and miliary disease.

\section{Pelvic MRI}

On the second day, Hedvig Hricak opened with Imaging of the Uterus, concentrating on the role of MRI. The prime role of MRI is as a problemsolving modality, which may obviate surgery. This imaging tool is especially valuable in müllerian duct anomalies, leiomyomata, and endometrial and cervical carcinoma. MRI distinguishes adenomyosis from leiomyoma, but does not differentiate degeneration in a myoma from sarcomatous change. Contrast-enhanced MRI is the modality of choice for endometrial carcinoma, and is far more accurate than ultrasound in this condition. MRI without contrast is the best modality for preoperative staging of cervix carcinoma. Pro-

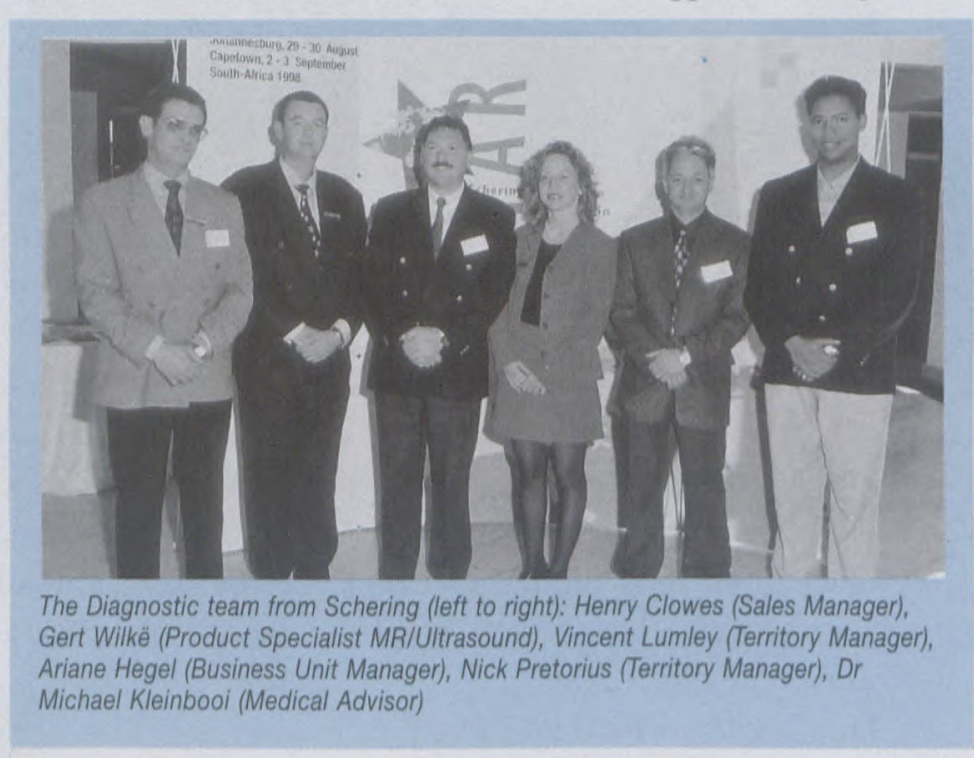
fessor Hricak emphasized that the tailored MRI examination is cost-effective when correctly used, and believes that the modality is misconstrued as a cost driver.

\section{Lung cancer imaging}

Elias Zerhouni followed with CT of lung carcinoma. He gave a thorough presentation of the TNM classification, with special reference to resectability criteria. He presented imaging characteristics of pulmonary neoplasms, with examples of carcinoma, metastases, carcinoid, etc. Regarding lymphadenopathy, CT is reported to have a bewilderingly wide range of accuracy, from $40 \%$ to $90 \%$. This highlights the limitations of cross-sectional imaging, although it is felt that the accuracy would improve should the short-axis diameter of the nodes be measured. The short axis is felt to be the most reliable parameter of nodal size. Further diagnostic improvements are expected in the next decade with the use of molecular markers. There is new evidence that aggressive surgical 


\section{SIEMENS}
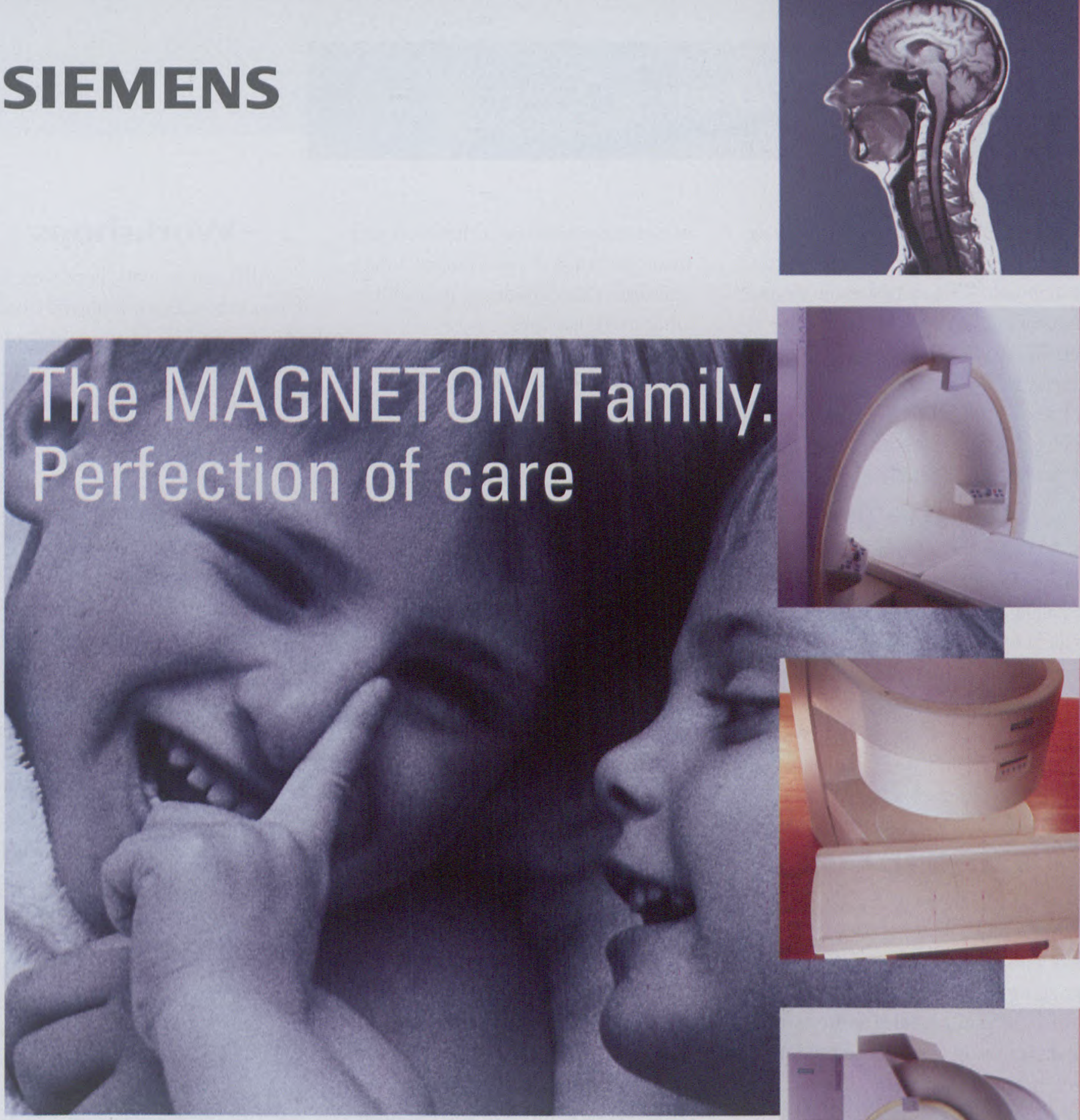

At the forefront of the constant evolution of medical technology, the challenge is to make the world of MR a better place for you and your patients.

The innovative MAGNETOM Harmony 1.OT and MAGNETOM Symphony $1.5 \mathrm{~T}$ is like music in the ears of the patient and doctor. They are the first new generation of compact magnets that do not compromise a $50 \mathrm{~cm}$ field of view.

The efficiency of the process is optimised by the use of the Integrated Panoramic Array ${ }^{\mathrm{TM}}$ which introduces a new coil system that saves time, ensures the patient's comfortability and sets the future standard.

These latest additions to the family come in attractive colours and the two machines share the same dimensions. This modular system can easily be upgraded which will last you well into the future.
The MAGNETOM Open viva $0.2 \mathrm{~T}$ is more comfortable in that it reduces patient claustrophobia and the attractive design warms the nervous patients up towards the therapy.

The MAGNETOM Vision plus, pushing the limits of MR. The redesigned MAGNETOM Vision plus remains the research flagship of our product family.

Siemens MRI - always staying advanced, saving time, controlling costs and improving confidence and comfort.

For more information contact:

Siemens Medical Engineering, Tel: (011) 652-2344, Fax: (011) 652-2322

E-mail:medical@siemens.co.za

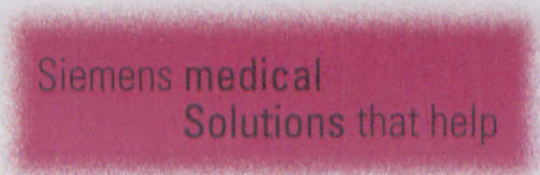

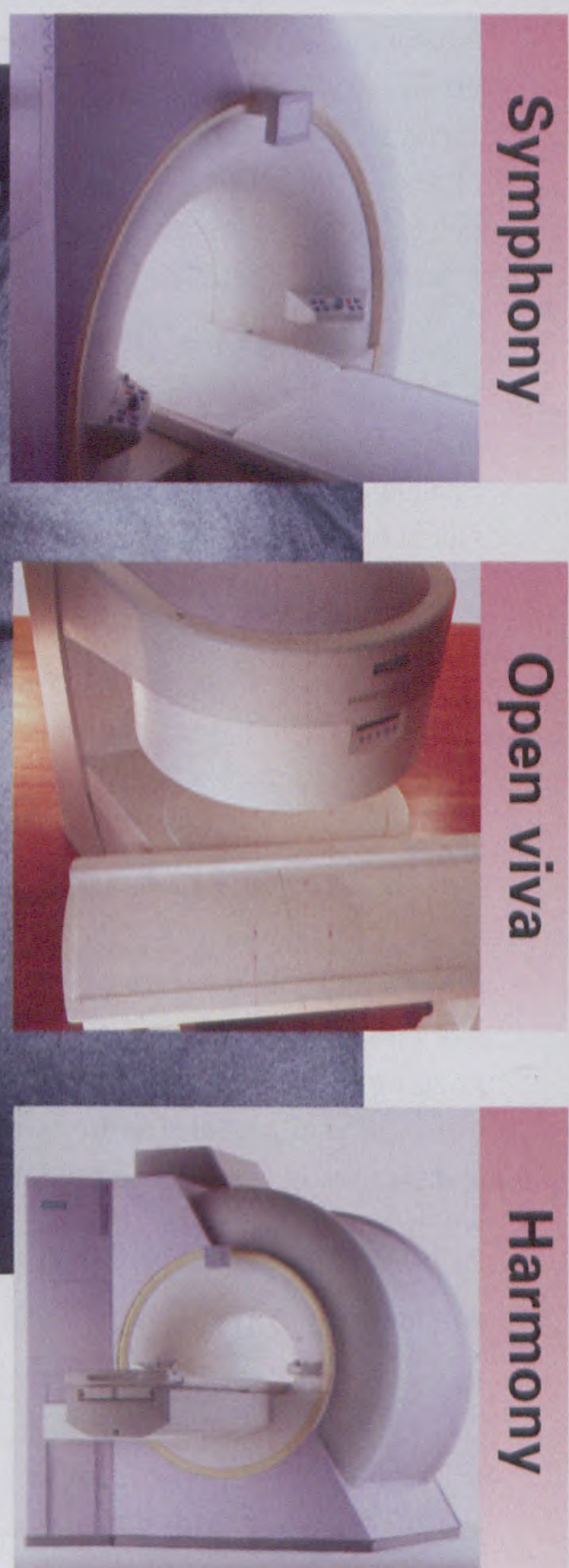

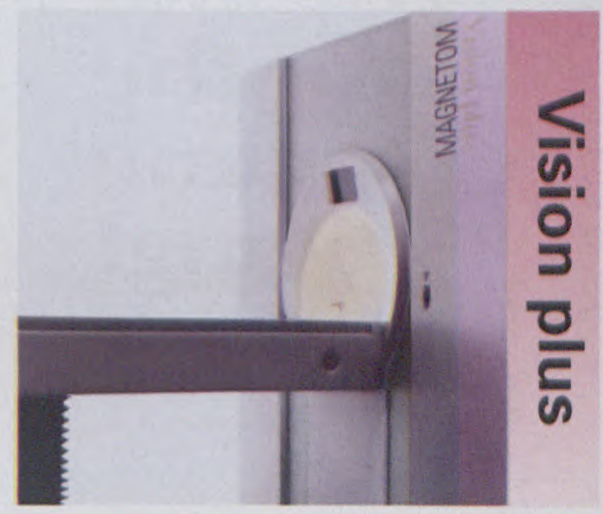




\section{from page 8}

surface coils produce images of diagnostic quality. For staging of prostate carcinoma, MRI surpasses ultrasound, but for guiding biopsies transrectal ultrasound is a reliable modality. MR spectroscopy further refines lesion location. The role of CT lies in assessing pelvic lymph nodes, where $0.7 \mathrm{~mm}$ is regarded as the threshold.

\section{Gl imaging}

Alexander Margulis then gave a comprehensive overview on Modern Imaging of the Alimentary Tube. The salient feature is the emergence of CT's application to the gastrointestinal tract. In small bowel obstruction, CT is the examination of choice, and enteroclysis is performed only if CT is negative. In the acute abdomen, CT is regarded as a cost-effective screening examination.

\section{Cardiac MRI}

This was followed by Advances in Cardiac MRI, by Elias Zerhouni. MRI has enormous potential in cardiac imaging, especially with innovative pulse sequences and echo planar imaging. Its use is not widely accepted yet, however, mainly because of inadequate software design. MRI has the potential to provide an integrated study of anatomy, mechanical function and tissue perfusion in one hour, including real-time cine sequences in multiple arbitrary projections.

\section{Renal imaging}

Hedvig Hricak then gave an absorbing lecture on Imaging of Renal Masses. A triphasic post-contrast $\mathrm{CT}$ technique is advocated. CT is vastly superior to ultrasound in detecting and characterising solid masses of $5 \mathrm{~mm}$ to $15 \mathrm{~mm}$ diameter, and is significantly superior to ultrasound even in the $15 \mathrm{~mm}-25 \mathrm{~mm}$ range. Ultrasound reliably distinguishes solid from cystic masses, but further characterization of solid lesions lies with CT and MRI. Contrast-enhanced MRI (with fat suppression) has slight superiority over spiral CT. Staging of carcinoma is best undertaken by $\mathrm{CT}$, but IVC thrombus is best detected by gradient echo MRI. MRI also demonstrates necrosis in lymph nodes. The recommendation is to reserve MRI for cases where CT is equivocal, for patients with renal failure and those at risk for a contrast reaction. Professor Hricak stated that a non-contrast spiral CT is now the primary examination for a suspected ureteric stone.

\section{Workshops}

After lunch, workshops were held. Elias Zerhouni's workshop on Conduct of CT and MR Examination of the Chest was superb. He gave a thorough consideration of imaging parameters, new advances allowing imaging at a pitch of six and a useful guide to contrast bolus location as predicated by the patient's pulse-rate. The advances in spiral CT technology will have farreaching implications for the future of routine imaging. MRI of the chest can be performed on most MR machines, and the technique was elucidated.

The meeting was very well organised. Congratulations and many thanks to the Schering team, especially Ariane Hegel and Ludwig Hahn. We are equally indebted to Jan Labuscagne, Congress Convenor of the Radiological Society, for his time and the considerable work he undertook to ensure the success of the meeting.

The STAR Program in South Africa was one of many that Schering have convened. One looks forward to a return visit to South Africa in the not toodistant future.

Vince Andrijich and Steve Beningfield

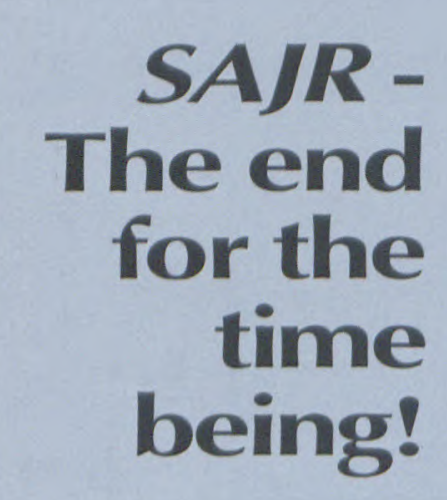

This edition of SAJR will be the last you will receive for a while. The Editors, Peter Corr and Steve Beningfield have performed miracles in providing the academic content, but in spite of their constant harassment of our colleagues, they have not received sufficient input from local radiologists to continue publication.

Our sponsors and advertisers have been magnificent, and the publisher Roger Short has worked tirelessly to produce a quality journal. Unfortunately that is not enough. Without the academic input the journal will not fly.

Compulsory continued professional development (CPD) starts next year. Publications will gain credits for their authors. We have pathology in abundance - who will take the gap and publish South African papers in a South African Journal?

SAJR is going into suspended animation while we wait for you to give it the kiss of life.

Richard Tuft

President, Radiological Society of South Africa 